\title{
THE METHOD ON GENERATING LAI PRODUCTION BY FUSING BJ-1 REMOTE SENSING DATA AND MODIS LAI PRODUCT
}

\author{
Jinling Song ${ }^{1}$, Jindi Wang ${ }^{1}$, Yueting Xiao ${ }^{1}$, Yanmin Shuai ${ }^{2}$, Wan Huawei $^{3}$ \\ ${ }^{1}$ State Key Laboratory of Remote Sensing Science; Beijing Key Laboratory for Remote Sensing of Environment and \\ Digital Cities; Center for Remote Sensing and GIS, School of Geography; Beijing Normal University, 19 Xinjiekou \\ Wai Street, Beijing, 100875, China. \\ ${ }^{2}$ Department of Geography and Environment; Center for Remote Sensing, Boston University, 675 Commonwealth \\ Avenue, Boston, MA. 02215, USA \\ ${ }^{3}$ Envi ronmental Monitoring Center, Ministry of Environmental Protection of the people' republic of China \\ songjl@bnu.edu.cn, wangjd@bnu.edu.cn, xiaoyueting2099@163.com, shuaiym@bn.edu, livelyhw@163.com
}

\section{Commission WG I/6}

\begin{abstract}
KEYWORDS: Vegetation; Computer Simulation; MODIS, Fusion; BJ-1, Remote Sensing, Priori Knowledge
\end{abstract}
\begin{abstract}
:
LAI (Leaf Area Index) is the more important parameter of vegetation canopy, which can depict their growth course. So LAI inversion from remote sensing observations is the hot study field, especially for the high spatial and high temporal resolution remote sensing data. Beijing-1 microsatellite is an applied earth observing microsatellite of China, which can also give us the good data of short cycle time and wider coverage. So it is necessary to generate the quantitative product of BJ-1 remote sensing data. In this paper, the main object is to study on the method of the leaf area index inversion for producing BJ-1 LAI product. Because of no on-board calibration for BJ-1 multi-spectral images, we can't get the reflectance data, but DN values. Therefore, we get the VI from BJ-1 multi-spectral data. And from analyzing some VIs, we take NDVI as the good index for the LAI estimation. In order to retrieve the leaf area index (LAI), the BRDF forward model is used to simulate the relationship between LAI and NDVI. Based on the BJ-1 LAI inversion, the second object of this paper is to generate of high spatial and high temporal resolution LAI product. A method is proposed to get high spatial and temporal resolution LAI product by fusing the time-series MODIS LAI product(1 km, 8-day product)and BJ-1 LAI. In this method, the BJ-1 classification image is used to register with MODIS data, then the percentage of classes of FPT classification in the MODIS pixel can be calculated, and the time-series LAI at every classes can be obtained through linear unmixing. At last, the BJ-1 LAI is used to adjust this curve of time-series LAI to estimate the LAI at high spatial and temporal resolution. Through this study, we can get the LAI products of BJ-1, which is with the high spatial resolution and high time resolution (32m, 4-day product). This product will provide more information of vegetation for BJ-1 microsatellite data applications.
\end{abstract}

\section{INTRODUCTION}

About $30 \%$ of the earth is covered by land and much of this is vegetated. Terrestrial vegetation impacts climate by playing a key role in various processes of energy, mass, and momentum exchanges. Remotely sensed hyperspectral data offer considerable potential for obtaining information about the earth surface. LAI(Leaf Area Index) is a biographic parameter determining vegetation photosynthesis, transpiration, and the energy balance of canopies(Bonan 1993). LAI(Leaf Area Index) is defined as one-side green leaf are per unit ground area in broadleaf canopies and as the projected needle leaf area in coniferous canopies. LAI is a key environment variable controlling an exchange of fluxex of energy, mass(e.g., water and $\mathrm{CO} 2$ ) and momentum between the Earth's surface and the atmosphere(Nikolay V. Shabanov 2005; Pandya 2006). And LAI is also the important parameter to descript the vegetation canopy structure and growing statues.

In this paper, the main object is to study on the method of the leaf area index inversion for producing Beijing-1 LAI product. Because of no on-board calibration for Beijing-1 multi-spectral images, we can't get the reflectance data, but DN values. Therefore, we get the VI from BJ-1 multi-spectral data. And from analyzing some VIs, we take NDVI as the good index for the LAI estimation. In order to retrieve the leaf area index (LAI), the BRDF forward model is used to simulate the relationship between LAI and NDVI. In this paper, the computer simulation model: Radiosity model is used, which can simulate the 3D realistic scenes of vegetation (such as crops and forest) in the whole growing period, and get their radiation regime. We can product the look up table of the vegetation structure parameters, sun direction, view geometry and the corresponding simulated spectrum. In addition, lots of measured data of structure parameters and spectrum for many types of vegetation is accumulated in China Typical Land Objects Spectral Database, which also can achieve the statistics relation ship of LAI and NDVI. So the look up table and measured data are taken as priori knowledge in LAI inversion of Beijing-1 images.

Based on the Beijing-1 LAI estimation, the second object of this paper is to generate the high spatial and high temporal resolution LAI product. One method is proposed to get high spatial and temporal resolution LAI product by fusing the time-series MODIS LAI product(1 km, 8-day product)and Beijing-1 LAI. In this method, the Beijing-1 classification image is used to register with MODIS data, then the percentage of classes of PFT classification in the MODIS pixel can be calculated, and the time series LAI at every land types can be obtained through linear unmixing. At last, the Beijing-1 LAI is used to adjust this curve of time series LAI, and to estimate the LAI at high spatial and temporal resolution. Through this study, we can get the LAI products of Beijing-1 images, which is with the high spatial resolution and high time resolution (32m, 4-day 
product), which will provide more information of vegetation for BJ-1 microsatellite data applications(Chen Zhengchao 2006).

In the past ten years, various medium resolution sensors have been launched. Several land products derived from these sensors, or will be soon available to the user community at both regional and global scales. Table 1.(Marie Weiss 2007).For almost 25 years, the Earth surface has been monitored at medium to coarse resolution (not better than $1 \mathrm{~km}^{2}$ pixels) thanks to the series of NOAA/AVHRR sensors. More recently, new sensors have been launched with improved spectral (e.g. SEAWIFS/VEGETATION/MODIS/MERIS) or directional (e.g. POLDER/MISR) sampling, and higher spatial resolution e.g. MERIS/MODIS).

\begin{tabular}{lllll}
\hline Reference & Project & Sensor & LAI & URL \\
\hline Knyazikhin et al.(1998) & NASA/MODIS & MODIS & 2000-now & http://cliveg.bu.edu/modismisr/laifpar/laifpar.html \\
Roujean and Lacaze & CNES/POLDER & POLDE & $1996-1997$ & http://polder.cnes.fr/ \\
(2002) & & R & 2003 & \\
Chen et al. (2002) & & AVHRR & $1993-2002$ & \\
Bacour et al. (2006) & ESA/MERIS & MERIS & $2002-$ now & http://www.brockmann-consult.de/beam/plugins.html \\
Baret et al. (2007) & FP5/CYCLOPES & VGT & $1998-2003$ & http://postel.mediasfrance.org/ \\
\hline Plummer et al. (2006) & ESA/GLOBCAR & VGT & $1998-2003$ & http://geofront.vgt.vito.be/geosuccess/ \\
& BON & ATSR & & \\
& & MERIS & $1998-2003$ & http://geofront.vgt.vito.be/geosuccess/ \\
& & ATSR & $1998-2003$ & \\
& & MERIS & $1998-2003$ & \\
\hline & VGT & $1998-2003$ & \\
\hline
\end{tabular}

The eight-day composites of the LAI/Fapar products (MOD15A2) version 4 pertaining to study sites were downloaded from the LP-DAAC Internet site. The MODIS $\mathrm{LAI} /$ Fapar product is produced at the 1-km spatial resolution daily(MOD15A1) and composited over an eight-day period, where the selected value in a compositing period is that with the highest corresponding Fapar. The products are projected on the integerized sinusoidal and sinusoidal 10o grid, respectively.

CYCLOPES products derived from VEGETATION sensors are available freely at the following web site: http://postel.mediasfrance.org/. The period 1998-2003 is currently available. Products are organized by tiles of $10^{\circ}$ zenith and $10^{\circ}$ azimuth. For effective use in such large-scale models, regional and global LAI must be available over a period of time. Field measurements of LAI, however, are cumbersome, time consuming, and impossible to obtain at the global scale. But satellite remote sensing is the most effective means of estimating LAI global fields on a regular basis(Pandya 2006). In this paper, the main object is to study on the method of the leaf area index inversion for producing BJ-1 LAI product. Based on the field measured data and computer simulation model simulated data, we can estimated the LAI of Beijing-1 images. Then according to the Beijing-1 LAI estimation, the second object of this paper is to generate of high spatial and high temporal resolution LAI product. A method is proposed to get high spatial and temporal resolution LAI product by fusing the time-series MODIS LAI product(1 km, 8-day product)and Beijing-1 LAI images.

\section{MATERIAL AND METHOD}

This section describes: 1) spectral and structure datasets of vegetations; 2) satellite data used in the study; 3 ) the generation of of Beijing-1 LAI maps; and 4) remote sensing data analysis and generation of LAI maps.

\subsection{Material}

Table.1 lists the all data used in this study. The field experiment sites are Shun Yi, Beijing,China and Luan Cheng, Heibei province, China. And the dates of Beijing-1 images imaging are 16 March, 06; 14 May, 06 and 17 June, 06 respectively. In order to get the time serial LAI variation, MODIS products (MOD15A2 and MOD12Q1) are used. Next we will introduce these data in detail.

\begin{tabular}{cc}
\hline Description & Site: Beijing \\
\hline Field experiment Sites & Shun Yi, Beijing,China \\
Location & Luan Cheng, Heibei \\
& province, China \\
& 16 March, 06 \\
& 14 May, 06 \\
Date of BJ-1 acquisition & 17 June, 06 \\
& 1 Jan 06- 31Dec,06 \\
Date of MODIS LAI & H26V4, H26V5, \\
8-day product & H27V5, H27V5. \\
MODIS Tile number & MOD12Q,2004 \\
MODIS Land cover product & (spatial resolution: 32m). \\
\hline BJ-1 multi-spectral image has green, red, NIR bands \\
\hline
\end{tabular}

Table.1 LAI Sites and details of satellite data/product acquisition used in the study.

\subsection{1. spectral and structure datasets of vegetations}

1) Field Sites and Experimental Measured data:

In the "Spectral Database System of Typical Objects in China" supported by 863 program, we have collected spectrums of typical objects and their corresponding parameters for about 20 years. In the database, there are more than 20,000 of the spectral data about vegetation, such as winter wheat, maize, rice, cotton and rapeseed, and also their corresponding growth structure parameters, the land use and land cover type in the experimental sites, crop phenology and farming methods(Wang Jindi 2004).

(a) Winter wheat data: winter wheat spectrum data and their parameters, LAI, LAD and so on in Shun Yi, Beijing in China in 2001,2003 and 2004;

(B) Maize data: maize spectrum data and their parameters LAI, LAD and so on in Luancheng, Heibei province in China in 
2000 and 2003.

2) Simulated datasets by computer simulation model

Because we don't have the experiment measured data for grass and forest, in this study, we use computer simulation model-Radiosity model, which can simulate the 3D realistic scenes of vegetation (such as crops and forest) in the whole growing period, and get their radiation regime(Song Jinling 2007(a); Song Jinling 2007(b)). We can product the look up table of the vegetation structure parameters, sun direction, view geometry and the corresponding simulated spectrum. And then the datasets of grass and forest are built by using the simulated data.

\subsubsection{Satellite Data Used in This Study}

1) Beijing-1 Microsatellite Multispectral images: Beijing-1(BJ-1) microsatellite is an applied earth observing microsatellite of China. It combines SSTL's standard Disaster Monitoring Constellation (DMC) multi-spectral camera with a high resolution panchromatic imager, which can achieve the $32 \mathrm{~m}$ spatial resolution multi-spectral images and the $4 \mathrm{~m}$ resolution panchromatic images synchronously. Beijing-1 images have the same characteristics and capabilities as TM image at 2、3、 4 bands. In addition, BJ-1 remote sending data can also give us the good data of short cycle time and wider coverage. So it is necessary to generate the quantitative product of $\mathrm{BJ}-1$ remote sensing data.

2) MODIS LAI product: The eight-day composites of the LAI/fAPAR products(MOD15A2) version 4 pertaining to study sites were downloaded from the Land Processes-Distributed Achieve Center(LP-DAAC) Internet site. The product was composited over an eight-day period, where the selected value in a compositing period is that with the highest corresponding Fapar. The products are projected on the integerized sinusoidal and sinusoidal 100 grid, respectively.

3) MODIS land cover product: the MODIS land cover product (MOD12Q1) version 4 was used (Table 1.) to investigate the biome assigned to the region of the study sites. The latest available MOD12Q1 product for our study period was 2004 and the product was used with the assumption that the biome distribution does not change with a year.

\section{2,2 METHOD}

\subsubsection{Beijing-1 LAI Map Generation}

In order to get the LAI distribution map at high spatial and high temporal resolution of Beijing-1 image, works were done shown in the flow chart (fig.1) :1)the spatial matchment of the Beijing image and MODIS products; 2) Generation of Beijing-1 LAI maps; 3) MODIS Products Processing; 4) The generation of time serials LAI of the whole year at Beijing-1 spatial resolution.

1) The image registration of Beijing image and MODIS products

Because MODIS products project is SIN project, which is different form BJ-1's projection, in order to match with BJ-1 image, MOIS products (MOD15A2 and MOD12Q1) are reprojected to the same projection with BJ-1 image, UTM projection. In this paper, we use the HDF-EOS and GCTP library in the actual dataset, which can provide coordinate transformation function, so can complete the conversion between the different projections easily.

Based on the coverage of the scene of Beijing- 1 image containing Beijing experiment sites, we select 4 tiles MODIS products (h26v04, h26v05, h27v04 and h27v05), accomplish the images Mosaic and then reproject them to UTM projection. Fig.2 is the reprojection image of MOD12Q1 product. According to MODIS PFT classification standard, the map is classified to the following classes: evergreen needleleaf trees, evergreen broadleaf trees, deciduous needleleaf trees, deciduous broadleaf trees, Shrub, Grass, Cereal crop, Broadleaf crop, Urban and built up and water. In this study, evergreen needleleaf trees, evergreen broadleaf trees, deciduous needleleaf trees and deciduous broadleaf trees are classified to one class, tree in the BJ-1 classification map(shown in fig.3), so in MOD12Q1 image, trees class represents evergreen needleleaf trees, evergreen broadleaf trees, deciduous needleleaf trees and deciduous broadleaf trees. Fig. 3 is the Beijing-1 classification map, which is classified to six classes: broadleaf crop, cereal crop, forest, grass, urban and water.

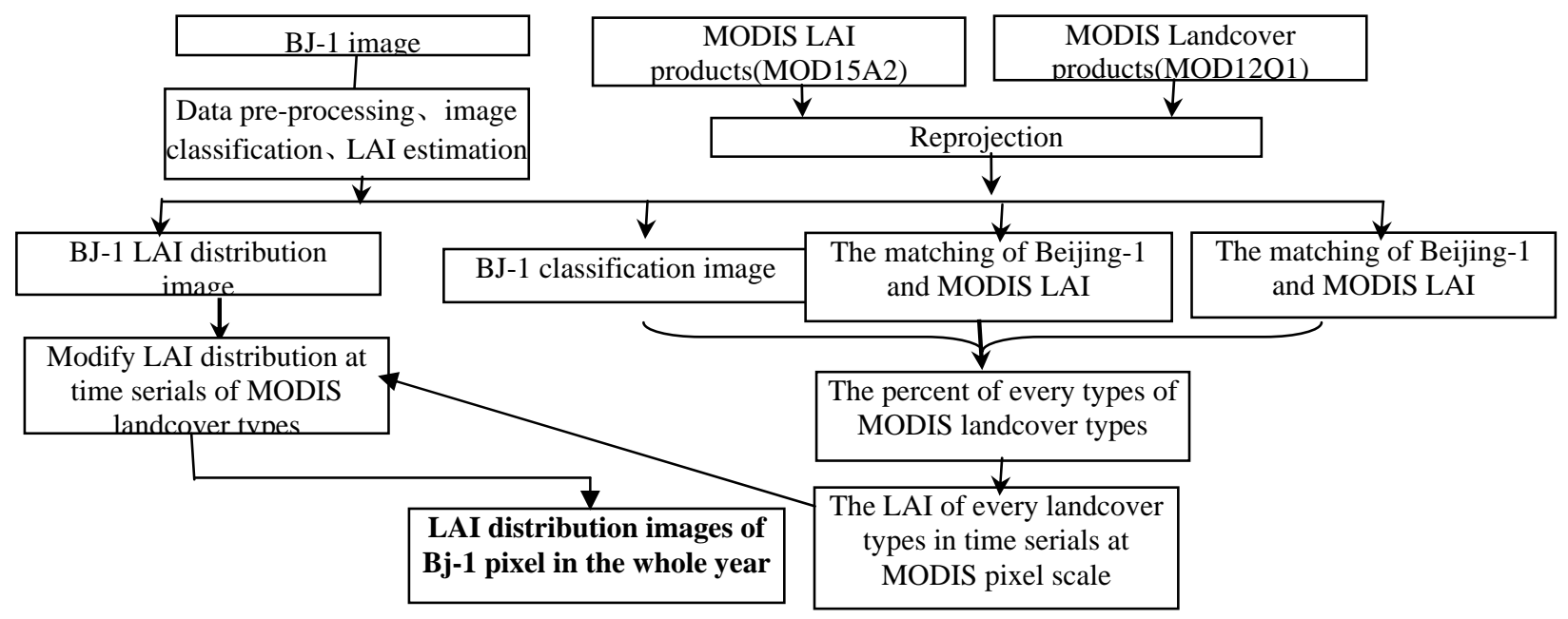

Fig. 1The flow chart of fusing MODIS and Beijing-1 image 


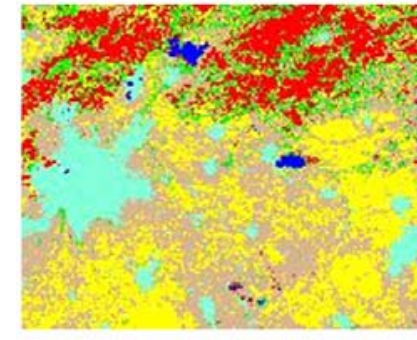

Fig.2 The reprojection image of MOD12Q1 product

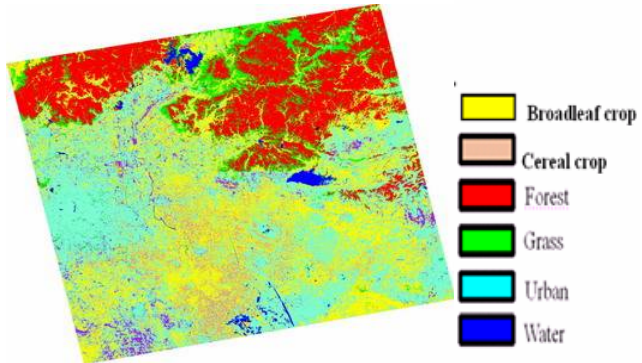

Fig. 3 The classification map of BJ-1 image
2) the generation of Beijing-1 LAI maps: LAI can be estimated at a variety of spatial scales and with different spaceborne sensors(Jing $M$. Chen 1996) using techniques ranging from regression models to canopy reflectance model inversions with varying success, which includes: (1) statistical models that relate LAI to band radiance(G. D. Badhwar 1986; Mehul R. Pandya 2006) or development of LAI-vegetation index relationships(Jing M. Chen 1996; Mehul R. Pandya 2006); (2) biophysical models like the Price model; and (3) inversion of canopy reflectance using a numerical model or LUT-based modeles(Mehul R. Pandya 2006). The empirical approach is common in remote sensing of LAI estimation. It is based on the idea that foliage reflectance is low in the red portion of the spectrum because most is absorbed by photosynthetic pigments, whereas much of the NIR is reflected by foliage. Then an empirical vegetation index such as normalized differences vegetation index(NDVI) based on the red and NIR is used to estimated LAI.

In this paper, we use the experiment data of Winter wheat in Shunyi, Beijing and maize in Luan cheng, in Heibei province. And the computer simulation model-Radiosity model is used to simulate the canopy regime of different LAI for grass and forest. Basic on these experiment data and the measured data, we can get the statistical relationship of NDVI and LAI, shown in Table.3. And using above relationship of NDVI and LAI, we can obtain the LAI distribution map of three periods: 16, Mar; 14, May; 17,Jun, shown in fig.4(Xiao Yueting 2008).

\begin{tabular}{||c|c|c||}
\hline Type & Formula & R2 \\
\hline Winter Wheat & NDVI=0.83*(1-exp(LAI/-1.155) $)$ & 0.623 \\
\hline maize & NDVI=0.9*(1-exp(LAI/-2.111) $)$ & 0.638 \\
\hline Grass & NDVI $=0.87 *(1-\exp (-\mathrm{LAI} / 0.873))$ & 0.961 \\
\hline Forest & $\mathrm{NDVI}=0.9 *(1-\exp (-\mathrm{LAI} / 1.702))$ & 0.564 \\
\hline
\end{tabular}

Table.3 The relationship of NDVI and LAI

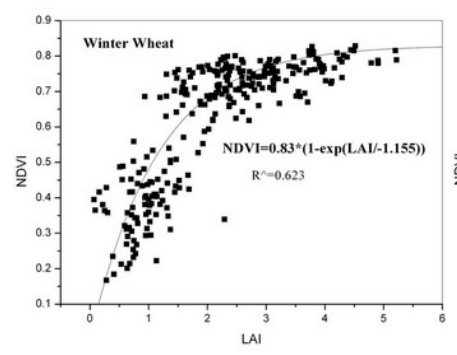

(a) Winter wheat

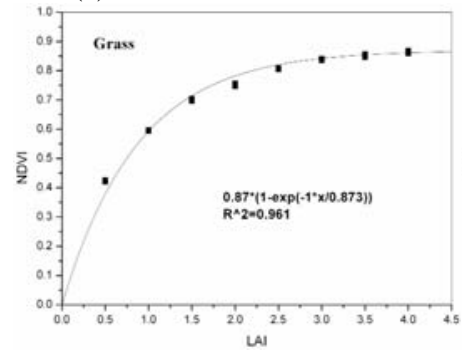

(c) Grass

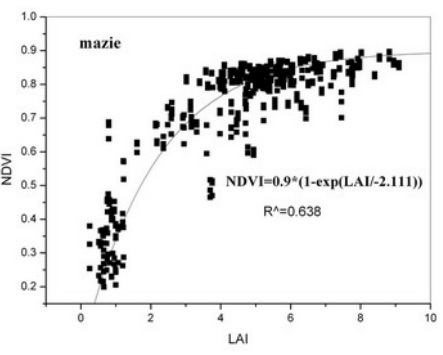

(b) Summer maize

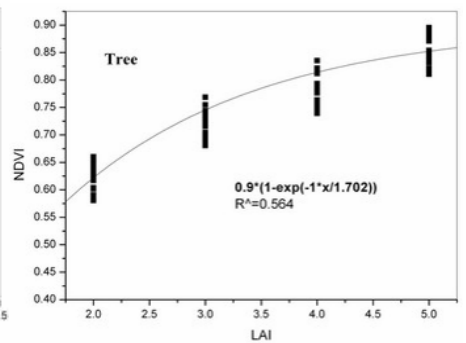

(d) Forest
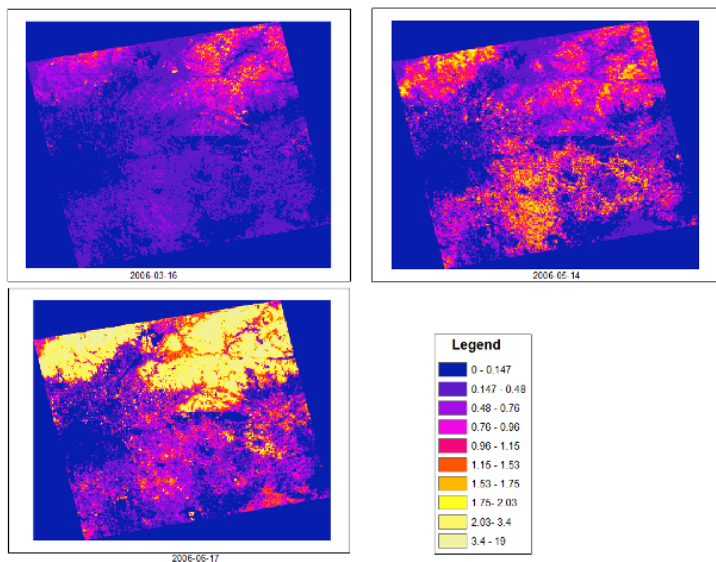

Fig. 5 The generation of LAI Map of Beijing-1 image(Xiao Yueting 2008)

Fig 4. The relationship of LAI and NDVI

\subsubsection{MODIS Products Processing:}

LAI can descript the vegetation growth, and in the growth time series, LAI variations of different land cover types have the significant difference. MODIS LAI products can offer stably time series LAI products, and can obtain the LAI variations of 
each land cover type in one year. The MOD15 LAI and fAPAR are 1-km products provided on a daily and eight-day basis. Where the globe is tiled into 36 tiles along the east-west axis, and 18 tiles along the north-south axis, are each approximately $1200 \times 1200 \mathrm{~km}$. A brief summary of the LAI algorithm is provided by Myneni(R. B. Myneni 2002). The algorithm is based on rigorous three dimensional radiative transfer (RT) theory(R. B. Myneni 1990). A lookup table (LUT) method is used to achieve inversion of the three-dimensional RT problem. The 250- and/or 500-m resolution bands are aggregated into normalized 1-km resolution grid cells prior to ingestion(R. E. Wolfe 1998).

In this study, based on different types of vegetation and the measured data and the simulated data, we can get the statistic relationship between LAI and NDVI, and based on the relationship, we can estimate the LAI of every pixel. And in this study, the quality of MODIS LAI has been analyzed: selected the pixels inversed LAI by the brief algorithm, and used the filter algorithm for pixels to get the available LAI inversion results(Xiao Zhiqiang 2008).

From the fig.2 and fig.3, we can see that two classification maps (MOD12Q1 and Beijing-1 image) match very well to a certain precision. Based on the two classification maps, the Beijing-1 classification map as the sub-pixels, we can get the percents of land types (forest, grass, cereal crop and broadleaf crop) in every MODIS pixels. Considering the percentage of for one land type more the $95 \%$, we can think this pixel is the pure pixel for one land type, and we can get the pixel counts of one land type (shown in fig.6). However, because of the coarse resolution of MODIS LAI products, $1 \mathrm{~km}$, and the pixels are most mixed, so in our research, we use pixel unmixing methods to get the LAI variation of every land cover types in time series at MODIS sub-pixels. The formula is shown as Equation 1.

$$
L=\sum_{j=1}^{n} f_{j} L_{j}+\varepsilon, \quad i=1,2,3 \cdots, m, \quad 0 \leq \sum_{j-1}^{n} f_{i} \leq 1
$$

Where, $L$ is LAI of mixed pixels, $f_{j}$ is percent of each land cover type in one pixel, $L_{j}$ is the LAI of $f_{j}$ percent land cover type, $\varepsilon$ is error. And selecting the mixed pixels, using the linear model decompose, then we can calculate LAI at sub-pixels by the Least Square method(Wan Huawei 2007). So extracting LAIs from the pure pixels of MOD15A2 product, we can get the statistic MODIS LAI in time serials of the whole year, shown in fig.7.

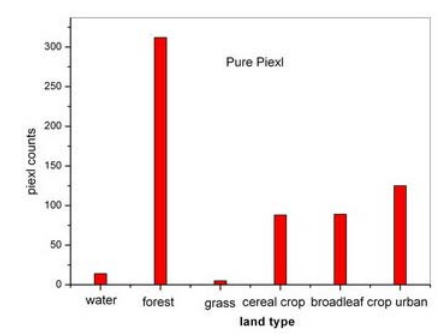

Fig. 6 The counts of pure pixels in MODIS product matched with Beijing-1 classification map

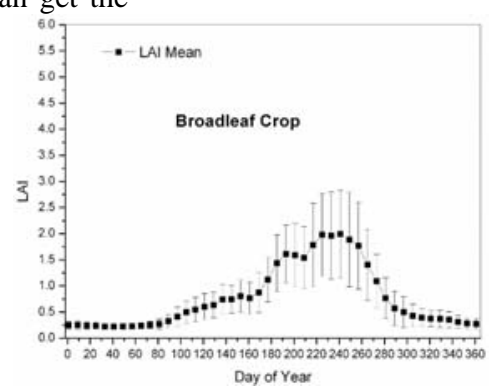

(a) Broadleaf crop

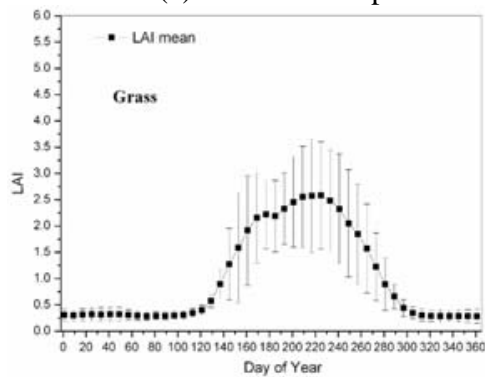

(c) Grass

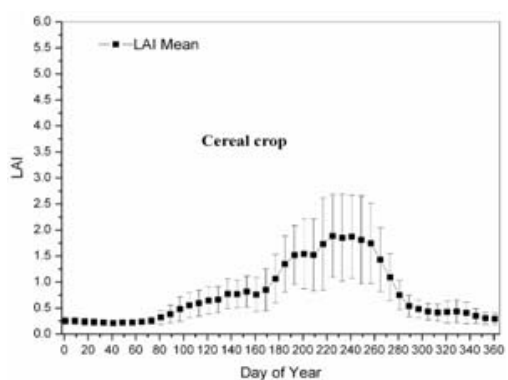

(b) Cereal crop

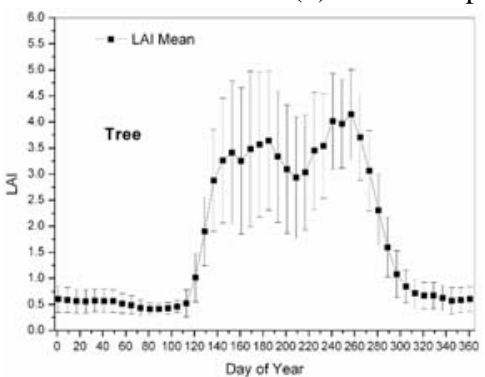

(d) Tree

Fig.7 MODIS Collection 42006 LAI trajectories for the reprojection MODIS LAI product: Means and one standard deviation values are shown.

\subsubsection{The generation of time serials LAI of the whole year at Beijing-1 spatial resolution}

And using the LAI estimation of BJ-1 image to modify the MODIS LAI Variation curve of every land cover types in one year, and in the course of modifying, we consider the error of BJ-1 LAI estimation and the error of MODIS LAI. There are three scenes of estimated LAI of Beijing- 1 images, which can be used to modify the LAI variation curve for every land types of MODIS. And then we can generate the time serials LAI (eight-day) of the whole year at Beijing-1 spatial resolution.

In this paper, the estimated LAI maps of Beijing-1 image in 75, 134 and 168 day of year are used, which are corresponding to the MODIS LAI in 73, 131, and 169 day of year. And the MODIS LAIs are considering as the background values, and the three scenes of estimated LAI of Beijing- 1 as the observed values to generate the time serial LAI of each land type. The method can be expressed by the Equation.2. In the formula, $n$ is the number of observed data; $r_{i}$ is the pixel, and $x_{b}$ is the background value,. In our study, the background value is the time serial MODIS LAI , and the $x_{o}$ is the estimated LAI of Beijing-1 image. $w\left(r_{i}, r_{j}\right)$ is the weight function, which can be valued as needed. Thus, we generate the time serials LAI (eight-day) of the whole year at Beijing-1 spatial resolution. 
And we show four scenes of generated LAI map in fig 8 , which are 57,145,177 and 209 day of yeae, respectively.

$$
x_{a}\left(r_{i}\right)=x_{b}\left(r_{i}\right)+\frac{\sum_{j=1}^{n} w\left(r_{i}, r_{j}\right)\left[x_{o}\left(r_{i}-x_{b}\left(r_{j}\right)\right)\right]}{\sum_{j=1}^{n} w\left(r_{i}, r_{j}\right)}
$$

\section{CONCLUSION AND DISCUSSION}

Because of the low spatial resolution, although MODIS LAI can give us time serial data, can't meet our study need. In this paper, based on the BJ-1 LAI estimation, fusing on time series
MODIS LAI products, and the BJ-1 LAI is used to adjust this curve of time-series LAI, we generate the high spatial and temporal resolution LAI product of Beijing-1 images. And this method is very useful for us to get high spatial and temporal resolution LAI product by fusing the data with low spatial resolution, but high temporal resolution and the data high spatial resolution, but low temporal resolution, and taking its advantages, respectively. So we can get the right data for our study. Through this study, we can get the LAI products of Beijing-1 images, which is with the high spatial resolution and high time resolution (32m, 4-day product). This product will provide more information of vegetation for BJ-1 microsatellite data applications. But this method has still in the course of testing, and next we would apply it to the larger area.

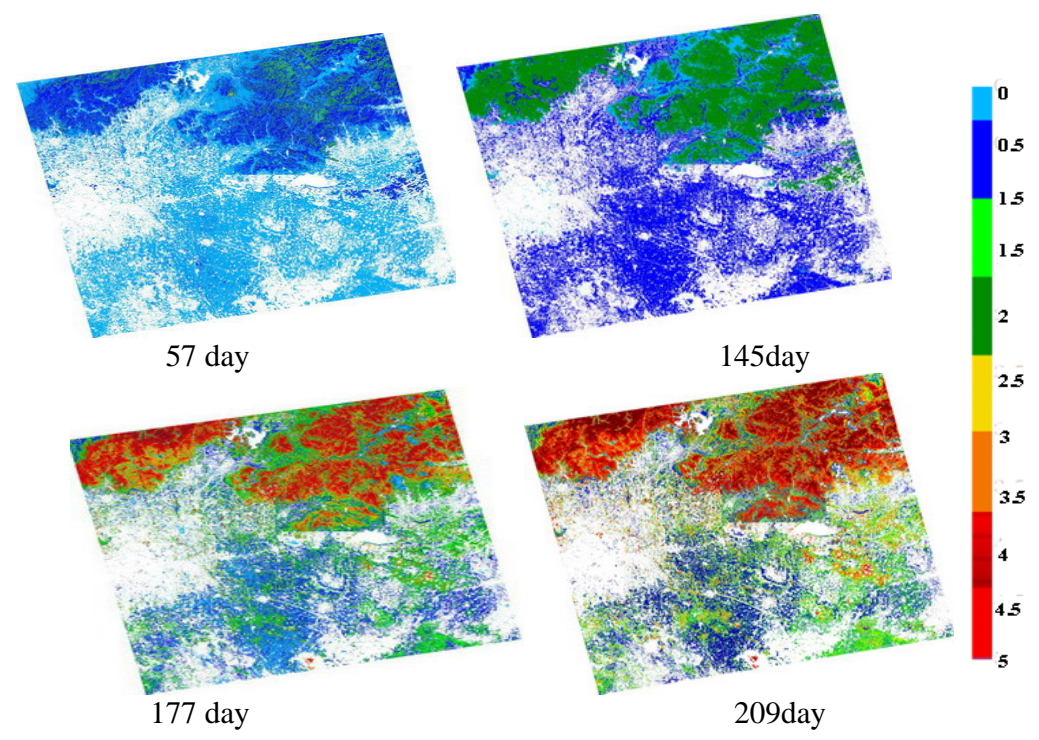

Fig. 8 The generation of time serial Beijing-1 LAI images.

\section{ACKNOWLEDGEMENTS}

We thanks for BLMIT supporting us free Beijing-1 images. This research was supported in part by the National Natural Science Foundation of China(No.40701116,40571107), the open fund about Beijing-1 image., and the Special Funds for Major State Basic Research Project (2007CB714407), National 863 Program (2002AA130010)

\section{REFERENCE}

[1] Bonan, G. B. (1993). Importance of leaf area index and forest type when estimating photosynthesis in boreal forests. Remote Sensing of Environment 43: 303-314.

[2] Chen Zhengchao, L. W., ZHANG Hao,L IU Xiang,ZHANG Liang (2006). The Geometric Evaluation of Be ijing-1 Microsatellite Multispectral Images. Journal of Remote Sensing 10(5): 690-696.

[3] G. D. Badhwar, R. B. M., N. C. Mehta (1986). Satellite-derived LAI and vegetation maps as input to global cycle models-A hierarchical approach. Int. J. Remote Sens 7: 265-281.
[4] Jing M. Chen, J. C. (1996). Retrieving leaf area index of boreal conifer forests using Landsat TM images. 50(2): 153-162.

[5] Marie Weiss, F. B., Sébastien Garrigues and Roselyne Lacaze (2007). LAI and fAPAR CYCLOPES global products derived from VEGETATION. Part 2: validation and comparison with MODIS collection 4 products. Remote Sensing of Environment 110: 317-331.

[6] Mehul R. Pandya, R. P. S., Karshan N. Chaudhari, Govind D. Bairagi, Rajesh Sharma,Vinay K. Dadhwal, and Jai Singh Parihar (2006). Leaf Area Index Retrieval Using IRS LISS-III Sensor Data and Validation of the MODIS LAI Product Over Central India. IEEE TRANSACTIONS ON GEOSCIENCE AND REMOTE SENSING 44(7): 1858-1865.

[7] Nikolay V. Shabanov, D. H., Wenze Yang, Bin Tan. (2005). Analysis and Optimization of the MODIS Leaf Area Index Algorithm Retrievals Over Broadleaf Forests. IEEE TRANSACTION ON GEOSCIENCE AND REMOTE SENSING 43(8): 1855-1865. 
[8] R. B. Myneni, G. A., and S. A.W. Gerstl (1990). Radiative transfer in three dimensional leaf canopies. Transport Theory Statist. Phys 19: 205-250.

[9] R. B. Myneni, S. H., Y. Knyazikhin, J. L. Privette, J. Glassy, Y.Tian, Y. Wang, X. Song, Y. Zhang, G. R. Smith, A. Lotsch, M. Friedl, J. T. Morisette, P. Votava, R. R. Nemani, and S. W. Running (2002). Global products of vegetation leaf area and fraction absorbed PAR from year one of MODIS data. Remote Sensing of Environment 83: 214-231.

[10] R. E. Wolfe, D. P. R., and E. Vermote (1998). MODIS land data storage, gridding, and compositing methodology: Level 2 grid. Remote Sensing of Environment 36: 1324-1338.

[11] Song Jinling, Wand Jindi., Liu Xiaoqing (2007(a)). Computer Simulation and Validation of BRF Data of Vegetation Canopy. Journal of Remote Sensing 11(2): 228-234.

[12] Song Jinling, Wang Jindi, Liu Xiaoqing, Wan Huawei (2007(b)). Computer Simulations of Differing Radiation Regimes on Aspen Trees. Journal of Remote Sensing 11(5): 670-677.

[13] Wan Huawei, Wand Jindi, Xiao Zhiqiang, Lili (2007). Generating the High Spatial and Temporal Resolution LAI by Fusing MODIS and ASTER. Journal of Beijing Normal University (Natural Science) 43(3): 303-308.

[14] Wang Jindi, L. X. (2004). The Spectrum Knowledge Base of Typical Objects and Remote Sensing Inversion of Land Surfaces Parameters. Journal of Remote Sensing 8(suppl): 4-7.

[15] Xiao Yueting, Wang Jindi, Song Jinling (2008). The Temporal Changing Information Extraction on Spectral Reflectance and Growth Parameters of Typical Crops. International Society for Photogrammetry and Remote Sensing(ISPRS): submited.

[16] Xiao Zhiqiang, W. J., Wang Zuosen. (2008). The generation and modification of MODIS LAI product in China. Journal of Remote Sensing in press. 
